\title{
PAULINA BOROWCZYK
}

Université Adam Mickiewicz, Poznań

\section{DE L'ÉQUIVALENCE À L'ADAPTATION}

Abstract. Borowczyk Paulina, De l'équivalence à l'adaptation [From equivalence to adaptation]. Studia Romanica Posnaniensia, Adam Mickiewicz University Press, Poznań, vol. XXXVI: 2009, pp. 33-54. ISBN 978-83-232-2035-0. ISSN 0137-2475.

The aim of this paper is to illustrate in which cases the translators use the adaptation when they are confronted with a term related to sociocultural aspects. We will discuss the notions of equivalence and adaptation and their limits in the translation. Some samples from Arte TV news and from the American film Shrek translated into Polish, German and French will be provided as a support for this article.

Comme le fait remarquer U. Dąbska-Prokop, ekwiwalencja to « pojęcie wieloznaczne i dyskusyjne, traktowane przez wielu teoretyków jako jedno z najważniejszych [...] pytań interesujących zarówno teorię przekładu, jak i praktykę oraz także dydaktykę tłumaczenia » (2000: 68). C'est ainsi que la notion de l'équivalence peut être cernée sur la base de multiples références, définitions ou opinions recueillies dans les différents travaux sur la traduction. L'étude de ces références permet de constater que l'équivalence peut être considérée aussi bien comme une «relation d'identité établie dans le discours entre deux unités de traduction de langues différentes, dont la fonction discursive est identique ou presque identique » (Delisle, 1999: 36), que comme un procédé de traduction, ponctuel ou global, consistant à remplacer un élément propre à la socioculture de départ par ce qui est propre dans la socioculture d'arrivée. Ce procédé de traduction que nous appelons dans ce qui suit l'adaptation, se situe au niveau extra-linguistique (référence aux mœurs, aux faits culturels, aux traditions propres à un pays donné...).

Les différentes acceptions du terme d'équivalence, qu'elles soient issues de la linguistique, de la stylistique comparée, de la traductologie ou de textes pragmatiques, seront discutées dans ce qui suit. D'abord, on verra comment les différents auteurs, linguistes et traductologues définissent l'équivalence. Ensuite, on observera que l'équivalence entre le TD et le TA s'établit à plusieurs niveaux, en fonction du cotexte, de la situation, du type du texte considéré, de sa fonction, etc. De plus, d'un 
point de vue pratique, on verra qu'à côté de l'équivalence globale s'établissant entre l'ensemble du texte original et le texte traduit (le traducteur transmet certains ressentiments ou images sans se soucier de l'adéquation des formes), il existe des équivalences ponctuelles résultant du fait que le traducteur, en traduisant un texte, le divise en plus petites parcelles pour pouvoir établir une équivalence entre un segment du TD et du TA (Lederer, 1994: 50). Finalement, on présentera la définition de l'adaptation pour pouvoir l'appliquer aux exemples provenant des journaux télévisés d'Arte et des versions doublées (française, polonaise et allemande) du film américain Shrek.

Vu qu'il n'existe pas d'équivalents absolus, il faut se contenter de la recherche des équivalents les plus proches et les plus naturels (the closest natural equivalent, Nida, 1964: 166). C'est ainsi que dans son ouvrage publié en 1964 Towards the Science of Translating, le linguiste américain et traducteur des textes bibliques E. Nida a distingué deux types d'équivalence : l'équivalence formelle et l'équivalence dynamique (1964: 159). La première

[...] focuses attention on the message itself, in both form and content. In such a translation one is concerned with such correspondences as poetry to poetry, sentence to sentence, and concept to concept. Viewed from this formal orientation, one is concerned that the message in the receptor language should match as closly as possible the different elements in the source language (1964: 159).

Il s'en suit que le traducteur transpose, dans le TA tous les éléments provenant $\mathrm{du} \mathrm{TD}$, sans en rien changer. Ce type d'équivalence exige du traducteur une comparaison permanente des deux textes afin d'évaluer le degré de l'adéquation du texte traduit et fait penser à la conception de Schleiermacher (1813) qui postulait qu'il faut «amener le lecteur à l'auteur » (Berman, 1984: 235). Comme le fait remarquer Nida, la traduction mot à mot peut être entre autres utile lors de la traduction des textes médiévaux. "Such a translation might be a rendering of some Medieval French text into english, intended for students of certain aspects of early French literature not requiring a knowledge of the original language of the text » (1964: 159). Ainsi, le texte traduit doit garder aussi bien la structure et la forme du texte original (syntaxe, expressions idiomatiques) que le contenu (thèmes et concepts).

Par contre, dans la traduction s'appuyant sur le principe de l'équivalence dynamique, il est indispensable de produire la même relation entre le récepteur et le texte d'arrivée que celle existant entre le récepteur et le texte original.

A translation of dynamic equivalence aims at complete naturalness of expression, and tries to relate the receptor to modes of behavior relevant within the context of his own culture ; it does not insist that he understand the cultural patterns of the source-language context in order to comprehend the message (Nida, 1964: 159).

Plus tard, la définition de la traduction que Nida donne ensemble avec C. Taber (1971) précise l'étendue de son application. Pour eux, la traduction doit « re- 
produire dans la langue réceptrice le message de la langue source au moyen de l'équivalent le plus proche et le plus naturel, d'abord en ce qui concerne le sens, ensuite en ce qui concerne le style (1971: 11, in : Hurtado-Albir, 1990: 79). Nida considère la Bible non comme un document mais comme un message qui doit parler à chaque lecteur d'aujourd'hui, sans avoir égard à sa langue, ni à sa culture. Sa théorie s'inscrit donc dans une dynamique de gommage des différences et de «couleur locale », visant avant tout à ne pas présenter d'obstacle à la compréhension. Ainsi, pour établir une équivalence entre les deux textes, il faut adapter le texte d'arrivée à la culture et à la langue connues des récepteurs pour qu'il ait le même effet que le texte de départ. Le principe de l'équivalence dynamique

vise donc autant que possible à dégager le message des contraintes formelles qui le lient à une langue, à une culture, à une époque historique particulière. Si le message, au bout de son voyage à travers le temps et l'espace, ne peut pas rester identique dans son apparence extérieure, il peut rester identique par la réponse qu'il suscite (Bensimon, 1998: 7).

Il en résulte que le texte traduit doit être compris immédiatement, sans hésitation d'aucune sorte. Pour illustrer le principe de l'équivalence dynamique, servons-nous de l'exemple évoqué par Ladmiral (1998) où il s'agit de la traduction du mot anglais pancake par crêpe en français ${ }^{1}$. Dans ce cas, le récepteur étranger n'est pas obligé de déchiffrer certaines allusions culturelles présentées dans le texte source car elles sont effacées dans le texte cible. Il importe peu qu'il les comprenne.

Dans ce cas, la traduction qui approche de la différence, de l'autre... fait disparaître la différence, réduit l'autre au même (à soi) : elle fait comme si elle était l'original, déliée de son contexte de départ; tout en prétendant conserver la fonction de TD en TA, elle modifie les présuppositions du texte pour les adapter aux nouvelles conditions de réception » (Gambier, 1992: 422).

Ce type d'équivalence fait penser à l'autre conception de Schleiermacher (1813), notamment celle où le traducteur «amène l'auteur au lecteur » (Berman, 1984: 235).

La notion d'équivalence devient aussi l'objet d'étude fondamental pour J. Catford (1965). Dans son ouvrage A Linguistic Theory of Translation, en déterminant

${ }^{1}$ Comme le dit Ladmiral, « [...] il arrive très souvent qu'à un item de langue source (Lo) il n'y ait pas à proprement parler d'équivalent exact en langue cible ( $\mathrm{Lt})[\ldots]$ Comment traduire en français le mot pancake? [...] un(e) pancake américain(e) n'est pas une crēpe bretonne, et encore moins une galette (au sarrasin) » (1998: 21-23). Cette absence d'équivalent est dûe essentiellement à son «irréductible singularité » (Bensimon, 1998: 10) d'une part. à son ancrage dans une culture de départ plus ou moins différente de la culture réceptrice de l'autre. C'est ainsi que l'allusion culturelle enferme le traducteur dans un dilemme: soit il préserve les termes étrangers et tend à garder «l'étrangeté de l'œuvre étrangère " (Berman, 1984: 17), soit il gomme l'allusion tout en garantissant une lecture facile du texte traduit, privé de l'exotisme. On voit là deux visées différentes, deux conceptions opposées qui affectent la traduction du culturel depuis des siècles et qui «débouchent sur des clivages polémiques essentiels» (Ladmiral, 1998: 24). 
la théorie de la traduction comme les relations entre le texte de départ et le texte d'arrivée, Catford la considère comme une branche de la linguistique comparée. «The theory of translation is concerned with a certain type of relation between languages and is consequently a branch of Comparative Linguistics [...]» (1965: 20). Et il définit la traduction de façon suivante: «the remplacement of textual material in one language (SL) by equivalent textual material in another language (TL)» (1965: 20). En traitant l'équivalence d'un phénomène empirique, l'auteur introduit une distinction entre textual equivalence et formal correspondence. "A textual equivalent is any TL text or portion of text which is observed on a particular occasion, by methods described below, to be the equivalent of a given SL text or portion of text $(1965: 27)$. A titre d'exemple, Catford cite une phrase anglaise qui est traduite en français :

\section{My son is six Mon fils a six ans.}

Pour pouvoir constater que les phrases sont équivalentes, on peut d'un côté demander l'opinion à un traducteur bilingue compétent. Ainsi, on peut répéter ce processus pour chaque élément dans le texte, p.ex. en demandant quel est l'équivalent français de $M y$ son dans le texte anglais, le traducteur fournit l'équivalent : Mon fils (1965: 27-28). De l'autre, on peut utiliser une autre procédure, appelée par Catford commutation. Elle consiste à introduire systématiquement des changements dans le TD afin d'observer des changements dans le TA qui résultent de cette opération. Dans ce cas, la définition de l'équivalence peut être modifiée : «that portion of a TL text which is changed when and only when a given portion of the SL text is changed » (1965: 28). Comme exemple peut servir l'échange de mon fils pour votre fille. Ainsi, «the TL text this time is Votre fille a six ans. The changed portion of the TL text (Mon fils / Votre fille) is then taken to be the equivalent of the changed portion of the SL text (My son / Your daughter) » (1965: 28). Il est pourtant à noter que les éléments sont interchangeables et équivalents seulement lorsqu'ils fonctionnent dans une situation comparable donnée, mềme s'ils n'ont que rarement la même signification au niveau linguistique. "The SL and TL items rarely have 'the same meaning' in the linguistic sens; but they can function in the same situation. In total translation, SL and TL texts or items are translation equivalents when they are interchangeable in a given situation » (1965: 49).

Par contre, d'après la définition de Catford, «a formal correspondent [...] is any TL category (unity, class, structure, element of structure, etc.) which can be said to occupy, as nearly as possible, the 'same' place in the 'economy' of the TL as the given SL category occupies in the SL $\gg(1965: 27)$.

Dans les années 70 et 80, les représentants de la Leipziger Schule (Otto Kade, Gert Jäger ou Albrecht Neubert) et de l'école Übersetzungswissenschaft provenant de l'Ouest de l'Allemagne (Wolfram Wilss, Katharina Reiss, Werner Koller) ont commencé à s'intéresser à la notion d'équivalence. Ainsi, les représentants de la Leipziger Schule ont considéré la traduction comme l'une des disciplines de la 
linguistique en l'appelant Translationslinguistik. Selon Kade, «alle Texte einer Sprache Lx (Quellensprache) können unter Wahrung des rationalen Informationsgehalts im Zuge der Translation durch Texte der Sprache Ln (Zielsprache) substituiert werden, ohne dass prinzipiell der Erfolg der Kommunikation beeinträchtigt oder gar in Frage gestellt wird » (1971: 26, in : Stolze, 1997: 56). Lors de la traduction, le changement des codes (Kodierungswechsel) a lieu mais le contenu informatif du texte que l'on transmet doit rester invariant.

Kade a aussi distingué quatre types d'équivalences potentielles pouvant s'établir entre le TD et le TA :

1. Die Eins-zu-eins-Entsprechung (totale Äquivalenz) - la correspondence s'établit au niveaux formel et sémantique entre deux langues, p.ex. (ang.) five (all.) fünf ; (fr.) bouc émissaire - (all.) Sündenbock ${ }^{2}$;

2. Die Eins-zu-viele-Entsprechung (fakultative Äquivalenz) - un terme possède plusieurs correspondants dans la LA, p.ex. (ang.) river - (fr.) fleuve / rivière ; (all.) Großvater - (suédois) morfar (de la part de mère) / farfar (de la part du père) ;

3. Die Eins-zu-Teil-Entsprechung (approximative Äquivalenz) - la relation dans laquelle pour un elément dans la LD tombe une partie d'élément dans la LA, p.ex. (fr.) esprit - (all.) Sinn, Geist, Verstand, Feinsinnigkeit ne sont que les correspondants partiels pour le terme français esprit ;

4. Die Eins-zu-Null-Entsprechung (Null-Äquivalenz) - elle a lieu lorsqu'il manque un correspondant dans la LA et concerne surtout les termes référant aux réalités socioculturelles (Realia-Bezeichnungen), p.ex. (all.) Berufsverbot - (fr.)? (1968: 79).

Cette division était surtout utile pour les textes spécialisés mais elle s'est avérée inadéquate pour la traduction des textes généraux. De plus, les types d'équivalences proposés par Kade concernaient avant tout la traduction des unités lexicales individuelles.

Par contre, l'autre représentant de la Leipziger Schule Neubert (1968, 1984, 1992) a surtout mis l'accent dans ses travaux sur les aspects pragmatique et textuel de la traduction. Pour lui, le texte traduit doit être adéquat du point de vue pragmatique, ce qui se reflète dans le choix des moyens stylistiques de la langue d'arrivée. Il a ainsi distingué deux types de traduction libre : « można [...] wyróżnić dwa rodzaje tłumaczenia wolnego, z których każde odpowiada normom semantycznym i gramatycznym języka przekładu, ale różnią się co do wartości pragmatycznych. Są to :

1) tłumaczenie wierne pod względem składników (die summandengetreue Übersetzung), które przejmuje relacje pragmatyczne języka źródłowego ;

2) tłumaczenie wierne pod względem postaci (die gestaltstreue Übersetzung), które zastępuje relacje pragmatyczne charakterystyczne dla języka źródłowego relacjami pragmatycznymi języka przekładu. Polega to na utworzeniu nowego

${ }^{2}$ Les exemples citẻs proviennent de l'ouvrage de Koller (1992: 229-232) qui a repris et développé la division de Kade. 
tekstu nie przez proste zastapienie środków gramatycznych i leksykalnych j1, stosując przy tym dodanie, przestawianie i opuszczanie elementów, ale przez rekonstrukcję pragmatyczną» (Neubert, 1968: 29, in : Kielar, 1988: 64).

Kielar nous en fournit un exemple. Si on traduit la formule allemande Rauchen verboten par Smoking prohibited en anglais, on reçoit un texte grammaticalement et lexicalement correct mais qui se soumet à la pragmatique du texte de départ. Mais la formule anlglaise No smoking est équivalente à la formule allemande non seulement aux niveaux grammatical et lexical mais elle surtout conforme à la pragmatique du texte d'arrivée (1988: 64).

En 1984, K. Reiss et H.J. Vermeer proposent une définition modifiée de l'équivalence. En partant d'une remarque générale que l'équivalence entre le TD et le TA a lieu à plusieurs niveaux du texte utilisé comme le moyen de la communication, ils constatent, ce qui nous paraît juste, que « die einzelnen Elemente auf den verschiedenen Ebenen können aufgrund der Verschiedenheiten der Sprachen und Kulturen in den meisten Fällen nicht invariant und nicht alle zugleich äquivalent gehalten werden » (1991: 169). Ainsi, le traducteur décide quels éléments du texte source sont fonctionnellement pertinents (le principe du choix) et dans quel ordre ils doivent être pris en considération dans le texte cible (le principe de la hiérarchisation). De même, il appartient au traducteur de décider

in welchen Fällen er sich für einen völligen Verzicht auf äquivalente Wiedergabe eines jeweiligen Merkmals entscheiden muss [...], und in welchen Fällen er sich für Kompensationen [...] oder für Reproduktion des Elements entscheiden muss, um für den Zieltext insgesamt Äquivalenz, d.h. Gleichwertigkeit in bezug auf die Funktion des Textes im Kommunikationsgeschehen innerhalb der Zielkultur zu erreichen (1991: 169).

Pour établir une relation d'équivalence entre le TD et le TA, le traducteur doit tenir compte des facteurs suivants : «der jeweilige sprachliche Kotext, der Situationskontext, die soziokulturelle Einbettung des jeweiligen Textes, die Zugehörigkeit des Textes zu einem Textyp und zu einer Textsorte [...] » (1991: 169-170).

Ainsi, « damit erweist sich Äquivalenz als dynamischer Begriff für eine Relation zwischen einem Ausgangs- und einem Zieltext, die bei jedem Text [...] eine Neuordnung der Relevanz aller jener Faktoren erfordert, die den Übersetzungsprozess beeinflussen " (1991: 170). C'est le traducteur qui choisit des moyens linguistiques convenables pour chaque texte à traduire afin d'établir une relation d'équivalence entre le TD et le TA.

En tenant compte des différentes opinions fournies par les différents traductologues (Nida, Nida et Taber, Catford, Kade, Jäger) au sujet de l'équivalence, W. Koller a proposé sa propre définition du terme :

Mit dem Begriff der Äquivalenz wird postuliert, dass zwischen einem Text (bzw. Textelementen) in einer Sprache L2 (ZS-Text) und einem Text (bzw. Textelementen) in einer Sprache L1 (AS-Text) eine Übersetzungsbeziehung besteht. Der Begriff Äquivalenz sagt dabei noch nichts über die Art der Beziehung aus : diese muss zusätzlich definiert werden (1992: 215). 
Et il la définit en distinguant cinq catégories qui déterminent l'équivalence d'ensemble. Ce sont :

1. Der außersprachliche Sachverhalt, der in einem Text vermittelt wird; den Äquivalenzbegriff, der sich am außersprachlichen Sachverhalt orientiert, nenne ich denotative Äquivalenz ;

2. Die im Text durch die Art der Verbalisierung [...] vermittelten Konnotationen bezüglich Stilsticht, soziolektale und geographische Dimension, Frequenz, etc. : den Äquivalenzbegriff, der sich an diesen Kategorien orientiert, nenne ich konnotative Aquivalenz;

3. Die Text- und Sprachnormen (Gebrauchsnormen), die für bestimmte Texte gelten : den Äquivalenzbegriff, der sich auf solche textgattungsspezifische Merkmale bezieht, nenne ich textnormative Äquivalenz ;

4. Der Empfänger (Leser), an den sich die Übersetzung richtet und der den Text auf der Basis seiner Verstehensvoraussetzungen rezipieren können soll, bzw. auf den die Übersetzung eingestellt wird, damit sie ihre kommunikative Funktion erfüllen kann ; die empfängerbezogene Äquivalenz nenne ich pragmatische Äquivalenz;

5. Bestimmte ästhetische, formale und individualistische Eigenschaften des AS-Textes : den Äquivalenzbegriff, der sich auf solche Eigenschaften des Textes bezieht, nenne ich formalästhetische Äquivalenz (Koller, 1992: 216).

Comme le fait remarquer Lederer

ces critères entrent inconstablement en ligne de compte, consciemment ou intuitivement, lorsqu'on juge une traduction mais ils ne sont pas pour autant des mêthodes de traduction [...] et ne permettront à quiconque d'apprendre à traduire, mais ils permettront à chacun de vếrifier s'il n'a pas, sur un point ou sur un autre, manqué à la nécessaire équivalence (1994: 55).

Il en résulte que ces critères permettent au traducteur de juger son texte d'arrivée par rapport à celui de départ.

Jusqu'ici, on a pu observer que la notion d'équivalence constituait l'objet d'intérêt et de discussion de beaucoup de chercheurs, surtout dans les années 19601990. D'abord, en ce qui concerne les approches linguistiques de la traduction, c'est Nida en 1964 qui a fait la disctinction entre l'équivalence formelle et dynamique. La conception de l'équivalence dynamique, quoique encore générale, avance sur le premier plan la reproduction du message source dans la langue réceptrice au moyen de «l'équivalent le plus proche et le plus naturel» (Nida, 1964: 166). Elle est un point de départ pour les notions proposées ultérieurement. Comme le fait remarquer Kielar

jej wartość polega jednak na tym, że otwiera drogę do przejścia do układów translatorycznych-typów, wyróżnionych według rodzajów tekstu podlegającego tłumaczeniu, aby ostatecznie zrealizować się $\mathrm{w}$ konkretnym układzie translatorycznym przez dialektyczne 
uwzględnienie szczególnych uwarunkowań danego procesu tłumaczenia $\mathrm{z}$ odniesieniem do uwarunkowań natury ogólnej (1988: 82).

Par contre, les translation equivalents de Catford (1965) sont des eléments textuels interchangeables seulement lorsqu'ils fonctionnent dans une situation comparable donnée, même s'ils n'ont que rarement la même signification au niveau linguistique.

Les représentants de la Leipziger Schule, et particulièrement Kade (1968), en postulant que le contenu de l'information à transmettre doit rester invariant, a distingué quatre types d'équivalences potentielles pouvant s'établir entre le TD et le TA. Ses propositions se rapportaient en réalité aux unités lexicales séparés et non à l'ensemble du texte.

Les propositions de Reiss et Vermeer (1984) et de Koller (1992) mettent en relief l'aspect textuel de l'équivalence. Les cinq catégories de Koller déterminant l'équivalence d'ensemble concernent plusieurs niveaux du texte sur lesquels s'établissent les relations d'équivalence entre le TD et le TA et dont le traducteur devrait tenir compte.

Dans ce qui suit, avant de passer à l'analyse de l'équivalence comme un procédé de traduction ponctuel, on voudrait démontrer comment est traitée la notion d'équivalence dans la pratique de la traduction. Comme le constate Lederer la notion d'équivalence est un élément central dans l'approche interprétative de la traduction. Et elle ajoute : «toutes les recherches effectuées à l'ESIT tendent à prouver que la traduction par équivalences a une validité générale quelles que soient les langues ou les types de textes [...] » (1994: 52). En distinguant la traduction par équivalences de la traduction par correspondances, on peut noter que cette première s'établit entre les textes et la seconde entre des éléments linguistiques, mots, figements ou formes syntaxiques (1994: 51). Pourtant, la visée interprétative n'exclut pas le recours aux correspondances à condition que celles-ci répondent aux besoins ponctuels. «Elle [l'équivalence] est le mode de traduction général, n'excluant pas pour autant les correspondances que justifie l'existence d'éléments qui correspondent en toutes circonstances : évocation hors contexte ou emploi dans un texte » (1994: 51). De plus, Lederer a distingué deux types d'équivalences : l'équivalence cognitive et affective qui doivent être considérées par le traducteur comme « un tout indissociable » dans le texte à traduire. Le premier type "découle de la jonction du sémantisme du texte et des compléments notionnels apportés par le traducteur » (1994: 52) tandis que le deuxième a de l'importance pour la traduction des textes littéraires. Le traducteur devrait ressentir l'émotion du texte de départ et l'exprimer pour le récepteur du TA.

De plus, comme nous l'avons signalé plus haut, à côté de l'équivalence globale s'établissant entre l'ensemble du texte original et le texte traduit (le traducteur transmet certains sentiments ou images sans se soucier de l'adéquation des formes), 
il existe des équivalences ponctuelles résultant du fait que le traducteur, en traduisant un texte, le divise en parcelles plus petites pour pouvoir établir une équivalence entre un segment du TD et du TA. Ces parcelles sont appelées par Lederer « unités de sens ». «Cette unité de sens est l'unité de traduction [...] pour laquelle on peut établir une équivalence dans une autre langue » (1994: 56). Ainsi, l'interprète ou le traducteur (ces unités de sens seront différentes pour le traducteur des textes écrits et l'interprète de simultanée) « établit une équivalence chaque fois que l'avancée des mots est suffisante pour que se produise une association entre leur sémantisme et des connaissances extra-linguistiques » (1994: 56). Cette opération peut concerner soit toute la phrase, soit un fragment plus long. Il se peut aussi que pour certains termes ou expressions, le traducteur trouve des correspondances. Il est difficile de dire exactement où commence et où finit l'unité de sens car celle-ci «n'a pas de longueur linguistique déterminée. Une idée ne se prête pas à mesurer, une équivalence non plus » (1994: 57).

Pourtant, comme le font remarquer Pisarska et Tomaszkiewicz, « jest to jednak trochę uproszczony obraz, bo mimo wszystko te tzw. « idee » są zawarte w formach i siłą rzeczy tłumacz ma tendencję do szukania czy tworzenia ekwiwalencji między jednostkami tekstu » (1996: 169). Et elles arrivent finalement à la constatation fondamentale qui s'applique à chaque type de traduction et avec laquelle on est d'accord : «ekwiwalencja jest pojęciem wieloznacznym, może dotyczyć jednostek o zmiennej długości, może sprowadzać się do porównywania form w kategoriach podobieństw i różnic, a może mieć charakter globalny i odnosić się do różnych ,informacji” zawartych w tekście » (1996: 169). Il s'en suit que les unités de traduction minimales peuvent varier et il est impossible de cerner précisément leur longueur ; elles dépendent largement de la façon dont le traducteur forme une équivalence entre le TD et le TA.

Contrairement à la notion d'équivalence discutée plus haut qui renvoie à la « relation d'identité établie dans le discours entre deux unités de traduction de langues différentes, dont la fonction est identique ou presque identique » (Delisle, 1999: 36), dans ce qui suit, l'objet du présent article sera l'équivalence en tant que procédé de traduction ponctuel ou global consistant à «remplacer une réalité socioculturelle de la langue de départ par une réalité propre à la socioculture de la langue d'arrivée convenant au public du texte d'arrivée » (Delisle, 1999: 9). Pour ce procédé de traduction nous utilisons également le terme d'adaptation.

Les deux linguistes et traductologues canadiens J. Vinay et J. Darbelnet dans leur ouvrage Stylistique comparée du français et de l'anglais (1966) ont fait la distinction entre l'équivalence et l'adaptation. Celles-ci appartiennent à deux des sept procédés auxquels se ramène la démarche du traducteur. Selon eux, l'équivalence consiste à rendre une expression figée, des clichés, des proverbes de la langue de départ par une expression figée, des clichés, des proverbes, qui « en mettant en œuvre des moyens stylistiques et structuraux entièrement différents » (1966: 52) dans la langue d'arrivée, rendent compte d'une même situation. Et ils illustrent 
l'emploi de l'équivalence sur l'exemple des proverbes : « like a bull in china shop : comme un chien dans un jeu de quilles » (1966: 52).

Toutefois, l'adaptation « $\mathrm{s}$ 'applique à des cas où la situation à laquelle le message se réfère n'existe pas dans LA, et doit être créée par rapport à une autre situation, que l'on juge équivalente. C'est donc ici un cas particulier de l'équivalence, une équivalence de situations » (1966: 52-53). Pour prendre un exemple, ils citent

le fait pour un père anglais d'embrasser sa fille sur la bouche comme une donnée culturelle qui ne passarait pas telle quelle dans le texte français. Traduire : he kissed his daughter on the mouth par il embrassa sa fille sur la bouche, alors qu'il s'agit simplement d'un bon père de famille rentrant chez lui après un long voyage, serait introduire dans le message LA un élément qui n'existe pas dans LD [...] Disons : il serra tendrement sa fille dans ses bras, à moins que le traducteur ne veuille faire de la couleur locale à bon marché (1966: 53).

Il est à voir que Vinay et Darbelent sont les partisans de l'adaptation lorsqu'ils constatent «le refus de procéder à des adaptations [...] se trahit dans un texte parafaitement correct par une tonalité indéfinissable, quelque chose de faux qui décèle invariablement une traduction » (1966: 53).

Pour citer un autre exemple, ils reprennent celui proposé par E. Nida. D'après lui, en traduisant la Bible «dans un pays où le figuier est considéré comme une plante nuisible, on adaptera la parabole du figuier en utilisant une autre plante " (1966: 5). Si on ne le faisait pas, le texte traduit serait perçu comme étranger et incomprêhensible.

L'objectif de l'adaptation proposée par Vinay et Darbelnet prétend que le texte d'arrivée peut atteindre les mêmes effets que le texte original sur les récepteurssource. C'est-à-dire qu'en préservant les mêmes effets communicationnels, le traducteur gomme les différences culturelles qui risquent de perturber la compréhension du texte cible. On peut alors se demander à quoi sert la traduction qui annule toutes les différences et fait disparaître les distances. Jusqu'où le traducteur peut-il aller dans sa pratique de l'adaptation ? Où en sont les limites? Et enfin, qu'est-ce que donner le message à une autre culture : est-ce changer la culture source ou bien la rendre plus proche du récepteur étranger? Pourtant, tout texte traduit est avant tout porteur et source d'un savoir sur la culture, l'histoire, la langue... de l'Autre. « La traduction n'est pas une simple médiation : c'est un processus où se joue tout notre rapport avec l'Autre» (Berman, 1984: 287). La compréhension de la différence culturelle est un apprentissage permanent de la tolérance et «une remise en question du propre système de références " (Lécrivain, 1998: 355). Pour qu'on puisse avoir accès à ce savoir, il est préférable de recourir plutôt à toute forme d'explication qu'à l'effacement des faits culturels.

La tendance à recourir aux différentes formes d'explication prouve que la chaîne de télévision franco-allemande Arte privilégie une démarche visant à montrer la différence culturelle et à tenter de la rapprocher des spectateurs du 
message cible et que les exemples où le traducteur substitue une réalité par une autre sont minoritaires dans le corpus analyse $e^{3}$. Par contre, on va montrer que dans les versions doublées du film d'animation Shrek, qui est un film de fiction ayant un caractère comique et ayant pour but de susciter une réaction concrète chez les spectateurs, notamment le rire, il y a beaucoup de cas où en se référant aux éléments socioculturels connus par les publics d'arrivée et en faisant allusion aux aspects caractéristiques pour les cultures cibles (p.ex. aux chansons, aux films, aux célèbres personnages du monde des médias, de la politique) les traducteurs donnent aux films traduits un caractère national et atteignent en même temps l'effet recherché, à savoir l'effet comique. Ainsi, d'un côté, on a affaire à la présentation des événements réels, des faits (le cas des JT d'Arte) où les traducteurs recourent rarement aux techniques adaptartices et de l'autre, il est question des histoires inventées qui sont riches en éléments plaisants et drôles où les traducteurs emploient beaucoup plus souvent le procédé d'adaptation. Dans le premier cas, on ne peut pas changer la réalité telle qu'elle est et dans le deuxième, il s'agit d'atteindre le même effet comique que dans l'original et de faire rire les spectateurs d'arrivée.

Selon Y. Gambier toutes les parties d'un texte n'atteignent pas le même degré d'adaptation (1992: 425). Ainsi, ce que nous avons montré plus haut, elle peut se situer aussi bien au niveau linguistique (les jeux de mots, les idiotismes, l'humour, le métalangage, les registres de langue...) qu'au niveau extra-linguistique (l'emploi des termes faisant référence aux différentes traditions, aux mœurs, institutions, partis politiques, événements historiques, artistiques...). Dans ce cas, l'adaptation est une tactique de traducteur dans des cas bien précis. Ne portant que sur certaines parties d'un texte, elle possède un caractère ponctuel. Par contre, il arrive aussi que le traducteur soit appelé à verser un texte d'un genre à un autre, comme p.ex. l'adaptation d'un roman en film, d'un poème en chanson, d'une pièce de théâtre pour la télévision, etc. Ce type d'adaptation que l'on appelle l' « adaptation globale » affecte la totalité du texte d'arrivée en élaborant un nouvel équilibre entre auteur et destinataires. Elle est «stratégique car elle restitue en priorité l'objectif global de l'auteur, c'est-à-dire sa visée » (Bastin, 1993: 478). J. Delisle, en parlant de l'adaptation globale, fournit un autre exemple ; «Dans la bande dessinée Tintin, les Dupont et Dupond deviennent les Thomson and Thompson (en anglais), les Hernández y Fernández (en espagnol) et les Schulze und Schultze (en allemand) » (1999: 9). Dans ce cas, les transformations auxquelles l'adaptation donne lieu sont de nature globale.

${ }^{3}$ L'analyse de la traduction des noms propres qui provenaient des journaux télévisés d'Arte enregistrés du 15 au 30 avril 2002 permet de constater que l'emploi de la démarche adaptative comprise comme un procédé de traduction ponctuel, est peu fréquente et non abusive. Elle est plutôt un moyen utile de rendre, de façon rapide, un terme propre à la langue et la culture de départ dans une langue d'arrivée et de produire le méme effet chez le destinataire. Sur 109 cas relevés dans notre corpus seulement $7 \mathrm{~d}$ 'entre eux étaient traduits au moyen de la technique d'adaptation. Cela constitue $6,4 \%$ de l'ensemble des exemples. 
Une perspective différente de l'adaptation est proposée par Bastin (1998), selon qui chaque type de transformation linguistique est menée dans le texte source afin d'atteindre des buts précis. Ainsi, au sens large du terme, l'adaptation impliquera une certaine liberté du traducteur à qui il serait permis d'introduire dans le texte cible toute la diversité des procédés, notamment les ajouts (l'explication, l'expansion...), les omissions, l'exotisation (les emprunts), la périphrase... (1998: 7, in : Dąbska-Prokop, 2000: 29). L'adaptation comprise comme ensemble de procédés pour véhiculer le sens, sera à la base de tout processus de traduction. Elle acceptera l'introduction de modifications pour que le récepteur étranger puisse comprendre le texte. Comme le remarque Bastin

il faut bien passer par l'adaptation si l'objectif est de faire passer le sens du message en produisant le mểme effet. L'adaptation est traduction. Ni moins, mais peut-être un peu plus, en ce sens qu'elle incarne et franchit un écart particulièrement grand entre deux réalités socio-linguistiques données et choisies. Selon l'ampleur de cel écart, la traduction sera l'adaptation [...] (1990: 474).

Suivant l'idée de M. Garneau, Y. Gambier préfere employer le concept et le terme de tradaptation car « il permet d'affirmer que toute traduction est adaptation » (1992: 425).

Ainsi, suivant la perspective selon laquelle l'adaptation est un terme général comprenant les différentes techniques de la traduction, on peut admettre qu'en traduisant un journal télévisé, le traducteur l'«adapte » à des récepteurs ayant des compétences linguistiques et socioculturelles différentes. Cette perspective disant que « toute traduction est adaptation» représente l'une des possibilités que l'on peut adopter pour désigner la phase de réexpression pendant laquelle le traducteur ajuste un message audiovisuel original à un contexte, à des spectateurs, à certaines intentions et visées... et le transforme à des niveaux linguistique, extra-linguistique, stylistique, visuel... Bastin considère même l'adaptation comme «le processus d'expression d'un sens visant à rétablir un équilibre communicationnel rompu par la traduction » (1993: 477). En employant les différents procédés de traduction, le traducteur «tradapte » le message audiovisuel original de sorte qu'il soit compréhensible pour les récepteurs étrangers. Dans le cas d'Arte, son objectif est d'offrir aux téléspectateurs un discours français ou allemand de sens équivalent à celui de départ, ce qui vaut, bien entendu pour chaque type de traduction.

Dans ce qui suit, en privilégiant le point de vue selon lequel l'adaptation est l'un des procédés de traduction ponctuels, on pourra observer qu'elle ne portera que sur certains segments du discours. Face à un mot ou un terme intraduisible, c'est-àdire désignant un élément du réel inconnu, et par conséquent n'ayant pas de nom dans la langue et la culture d'arrivée, le traducteur peut adapter comme équivalent de sens un terme désignant la chose la plus comparable dans la langue et la culture du destinataire. En principe, il s'agit de déclencher, dans la tête du récepteur étranger, le même type d'associations qu'évoque l'original. Ainsi, ce qui est propre 
à la culture source est substitué par ce qui est caractéristique de la culture cible. C'est donc à l'adaptation au niveau extra-linguistique que nous aurons affaire dans les exemples qui suivent.

Les exemples qui suivent montrent comment un élément de la culture source est substitué par un élément de la culture cible. Ils illustrent le type d'équivalence appelé par Kade «die Null-Äquvialenz » (1968: 79, in : Koller, 1992: 232). Elle a lieu lorsqu'il manque un correspondant dans la LA et concerne surtout les termes qui référent aux réalités étrangères. Nous commenceons notre analyse par les exemples tirés des JT d'Arte pour ensuite passer à la comapraison des cas provenant des versions doublées du film américain Shrek.

Etant donné l'incompabilité des systèmes scolaires français et allemands, le traducteur décide dans les cas qui suivent d'utiliser dans les textes d'arrivés, un terme connu du public cible, remplissant une fonction pareille au terme employé dans le texte de départ. «Tłumacz korzysta z tej strategii, jeśli zamiast realiów występujących w tekście wyjściowym szuka słów, które dla publiczności kultury docelowej będą miały identyczną albo przynajmniej podobną zawartość informacyjną i/lub skojarzeniową i emocjonalną» (Valló, 2002: 76, in : Jarmołowicz, 2004: 115). Voici les exemples illustrant la dếmarche adaptative :

\begin{tabular}{|c|c|}
\hline llemand (Texte de départ) & Texte français (Texte d'arrivée) \\
\hline $\begin{array}{l}\text { 1 } \\
\text { Philippe Guilpain (professeur) : } \\
\text { - "Le professeur d'allemand, il faut/lui, c'est le } \\
\text { seul qui doit aller chercher ses elèves. Alors que } \\
\text { les autres, ils savent à la rentrée qu'ils auront } \\
\text { des elèves. Bon, le professeur d'allemand, lui, } \\
\text { il faut qu'il aille les chercher. Il faut que tout } \\
\text { au long de l'année qu'il s'investisse pour } \\
\text { aller/pour convaincre les futurs sixièmes de } \\
\text { prendre l'allemand au lieu de prendre anglais. } \\
\text { Voila, c'est toute la stratégie» }(18.04 .02)\end{array}$ & $\begin{array}{l}\text { - «Als Deutschlehrer muss man sich um seine } \\
\text { Schüler bemühen. Die anderen Lehrer wissen } \\
\text { dass sie genügend Schüler haben werden, der } \\
\text { Deutschlehrer nicht. Das ganze Schuljahr muss } \\
\text { man sich dafür einsetzen dass die zukunftigen } \\
\text { Fünftklässler Deutsch statt Englisch wählen. } \\
\text { Das ist die Strategie». }\end{array}$ \\
\hline $\begin{array}{l}\text { Images + commentaire off: } \\
\text { - "Il doit aussi convaincre les parents. Dès le } \\
\text { deuxième trimestre de l'annee scolaire qui } \\
\text { précède l'entree au collège. il ècrit à chacun } \\
\text { d'eux pour présenter tous les avantages qu'ils } \\
\text { ont à choisir l'allemand en première langue " } \\
(18.04 .02)\end{array}$ & $\begin{array}{l}\text { " « Er muss auch die Eltern überzeugen. Im ersten } \\
\text { Halbiahr der vierten Klasse schreibt er allen } \\
\text { Eltern einen Brief und erklärt ihnen die Vor- } \\
\text { teile, die es mit sich bringt, Deutsch als erste } \\
\text { Fremdsprache zu wählen ». }\end{array}$ \\
\hline
\end{tabular}

Dans le premier exemple le groupe nominal propre au système scolaire français, à savoir les futurs sixièmes est remplacé par un équivalent allemand die zukunftigen Fünftklässler. Après avoir terminé l'école élémentaire que les élèves 
français quittent au bout de 5 ans, ils changent d'établissement pour se diriger vers le collège. A l'âge de 10-11 ans, ils entrent alors en sixième (cycle d'orientation) et doivent passer des tests nationaux qui visent à vérifier le niveau de leurs connaissances. Le changement de classe marque donc le passage d'une école à l'autre. Pour produire le même effet chez le spectateur allemand, le traducteur s'est servi d'un groupe nominal die zukunftigen Fünftklässler désignant les élèves allemands qui après avoir quitté l'école primaire (Grundschule) au bout de 4 ans, sont orientés à l'âge de 9-10 ans vers différents types d'établissements scolaires : la Hauptschule, la Realschule, le Gymnasium ou la Gesamtschule. Dans chacune de ces écoles, les elèves allemands passent d'abord deux ans dans des classes d'orientation (Orientierungsstufe), puis ils sont orientés en fonction des notes et des goûts vers une des quatre filières. Les élèves de la cinquième (die Fünftklässler) sont donc au premier degré d'orientation. En observant l'original et sa traduction, on peut remarquer que le numérotage des classes differe dans les deux pays. En France, les chiffres descendent à partir de la sixième (le début du collège) à la première et aboutissent à la terminale tandis qu'en Allemagne, ils augmentent de la première (on commence à compter dès l'école primaire) à la treizième. Malgré les différences entre les systèmes français et allemand et entre les âges qu'ont les élèves lorsqu'ils passent d'une école à l'autre, le traducteur vise à atteindre une identité de sens consistant à accentuer le moment de changement d'école et le fait de devoir choisir à ce moment-là une langue étrangère.

Avant que ses élèves entrent au collège, le professeur doit aussi persuader leurs parents. Pour le professeur d'allemand, qui est une exception en comparaison des autres professeurs de langues étrangères dans cette région, toute la procédure commence déjà à la dernière classe de l'école primaire. Dans le deuxième cas, le traducteur a procédé par l'emploi de deux adaptations dans le TA. Les éléments propres au système scolaire français, notamment la période précédant l'entrée au collège et la division de l'année scolaire française en trimestres ont été susbstitués par des eléments propres au système scolaire allemand. Par conséquent, on retrouve dans le texte cible la quatrième (die vierte Klasse) qui est la dernière classe de la primaire en Allemagne et précède l'entrée dans une école secondaire. De plus, contrairement à la France, en Allemagne, l'année scolaire est divisée en deux semestres (das Halbjahr). Ainsi, le début du deuxième trimestre en France (après Noël et le Nouvel An) correspond au premier semestre allemand.

Le procédé de l'adaptation qui est axé sur l'efficacité de la communication et sur l'adéquation aux attentes du récepteur, "fige l'image que l'on se donne du savoir et de la préparation intellectuelle du public dans sa capacité d'ouverture aux pratiques langagières et culturelles autres » (Lécrivain, 1998: 352). Comme le remarque $\mathrm{C}$. Lécrivain, le fait que certains allusions ou éléments culturels échappent au lecteur d'un texte source n'est pas considéré comme obstacle de la part de l'auteur tandis que la lecture du texte traduit laisse peu de place au rationnel, à la déduction ou l'intuition du lecteur. 
La lecture du texte traduit, tout comme celle d'un texte original, est un acte d'intelligence où se développe pleinement le processus de la connaissance, et généralement le contexte possède suffisamment d'indications pour que le lecteur rétablisse à peu près convenablement ce qui pouvait lui paraître anormal, étrange ou déroutant. La participation du lecteur est une participation dynamique et l'objet imaginaire se constitue à partir d'une série de combinaisons, d'associations, de compositions, etc., qui aboutissent à l'interprétation figurative. Ce processus de la connaissance n'est pas nécessairement immédiat, linéaire, progressif et fluide (Lécrivain, 1998: 353).

Nous tenons à souligner que nous partageons l'avis de Lécraivain quant à la traduction présentée sous forme écrite. Pourtant, dans le cadre audiovisuel, où le spectateur est confronté à la rapidité de réception des messages et par conséquent, à l'évanescence des mots et des images, la saisie du sens doit se produire immédiatement, au moment de la réception. À l'écrit, elle peut avoir lieu à différents moments puisqu'on peut relire, revenir en arrière, etc... Dans les médias, cela n'est pas possible. Les différences se situent aussi bien au niveau des mécanismes mis en place pour faire comprendre l'écrit ou l'oral et le visuel, qu'au niveau des compétences que l'on exige de l'individu. C'est pour cela, pour que la saisie du sens puisse avoir lieu immédiatement, que le traducteur remplace parfois «une réalité socioculturelle de la langue de départ par une réalité propre à la socioculture de la langue d'arrivée convenant au public cible du texte d'arrivée » (Delisle, 1999: 9).

Dans ce qui suit, nous étudierons les cas où le traducteur procède par la substitution des établissements à caractère administratif et juridique appartenant à une socioculture donnée par les organismes jouant un rôle comparable dans la socioculture d'arrivée.

Dans le troisième exemple, le traducteur a remplacé le substantif français la mairie par le substantif allemand das Rathaus.

\begin{tabular}{|l|l|}
\hline \multicolumn{1}{|c|}{ Texte français (TD) } & Texte allemand (TA) \\
\hline 3 & \\
Images + commentaire off : & \\
- «Hier soir, ici plus qu'ailleurs, on a sabré le & • "Hier knallten die Champagnerkorken gestern \\
champagne. La mairie de Marignane, c'est un & Abend lauter als anderswo. Marignane ist von \\
des fiefs de l"extrème droite française. Dans & jeher eine Hochburg der französischen Rechts- \\
cette bátisse, Daniel Simonpiéri régnant maître, & radikalen. Im Rathaus thront Daniel Simonpiéri \\
il a gagné la ville lors des municipales de & als uneingeschränkter Herscher seit den Kom- \\
$1995 »(22.04 .02)$ & munalwahlen von 1995». \\
\hline
\end{tabular}

Les bâtiments français et allemand où se trouvent le bureau du maire (Bürgermeister en Allemagne) et les services de l'administration jouent un rôle similaire dans les deux pays. Ainsi, sans avoir voulu introduire un emprunt dans le texte cible, le traducteur a préféré employer un terme connu des spectateurs allemands. On peut supposer que das Rathaus déclenche le même type d'associations chez un Allemand que la mairie chez un Français. C'est aussi bien un siège du maire qu'un 
bâtiment où on déclare un mariage, un divorce, une naissance, un décès ou on délivre des documents administratifs...

Comme le fait remarquer Valló qui s'occupe de la spécificité de la traduction des drames, «strategia ta jest dowodem na to, że przy pragmatycznych operacjach przekształcających decydująca rolę odgrywają aspekty kulturowe, a nie językowe » (Valló, 2002: 76, in : Jarmołowicz, 2004: 115). Pourtant, en se servant de ce procédé, le traducteur doit faire attention pour ne pas perdre complétement la couleur locale du texte de départ et ne pas déformer le caractère du texte et de la situation présentée dans ce texte. "Adaptacja pragmatyczna kryje w sobie niebezpieczeństwo przeniesienia do tekstu docelowego takich zmian treściowych i stylistycznych, które są sprzeczne z duchem języka wyjściowego » (Valló, 2002: 76, in : Jarmołowicz, 2004: 115). Mais ce n'est qu'au niveau verbal que l'adaptation peut avoir lieu à la télévision ou au cinéma. L'image restant intacte montre la couleur locale et le monde réel de l'Autre. De plus, il nous semble que la démarche adaptative employée dans les cas déjà cités et dans celui qui suit, garde son caractère ponctuel, ce qui permet au spectateur d'arrivée de suivre sans interruption la suite de l'actualité et sans devoir réfléchir à quelle réalité renvoie le terme étranger. Ainsi, l'application de l'adaptation comme un procédé ponctuel importe sur la bonne et rapide réception de la totalité du message.

Dans l'exemple suivant, le nom d'un organe central de police allemand a été substitué par un terme analogue existant dans la réalité française :

\begin{tabular}{|c|c|}
\hline Texte allemand (TD) & Texte français (TA) \\
\hline $\begin{array}{l}4 \\
\text { Images + commentaire off : } \\
\text { - " Eine weitere Frage steht im Raum : hatte das } \\
\text { Bundeskriminalamt Terrorwarnung? Die Zeit- } \\
\text { ung Bild behauptet das heute, das Innenmi- } \\
\text { nisterium dementiert allerdings heftig. Richtig } \\
\text { ist aber auch : Beamte des BKA hielten sich in } \\
\text { den Tagen vor der Explosion auf Djerba in } \\
\text { Tunesien auf, wohl weil die Situation dort für } \\
\text { gefährlich gehalten wurde »(17.04.02) }\end{array}$ & $\begin{array}{l}\text { - « Autre question : la police iudiciaire était-elle } \\
\text { au courant d'un attentat imminant, comme l'af- } \\
\text { firme aujourd'hui le journal populaire allemand } \\
\text { Bild? Le Minisitere de l'Intérieur a démenti } \\
\text { l'information. On sait aujourd'hui que quelques } \\
\text { jours avant l'explosion, les policiers allemands } \\
\text { se trouvaient en Tunisie, sans doute parce que la } \\
\text { situation avait été jugée dange-reuse». }\end{array}$ \\
\hline
\end{tabular}

Ici, le traducteur a trouvé dans le texte cible un équivalent (la police judiciaire) d'un substantif allemand das Bundeskriminalamt pour désigner un organe de police qui coordonne, au niveau national, la lutte contre le crime. Appartenant à des réalités différentes, aussi bien les policiers et les fonctionnaires du BKA que ceux de la P.J. sont chargés de poursuivre, rechercher et arrêter les auteurs d'infraction. Leurs actions ont pour but commun de réprimer les délits. Ainsi, en étant confronté dans le texte de départ à un élément de réel inconnu qui n'a pas de correspondant exact dans la langue et culture cibles, le traducteur a adopté comme équivalent la chose la plus comparable dans le texte du destinataire. 
Dans les exemples étudiés, on a pu observer que les différences entre deux réalités socioculturelles (l'incompabilité des systèmes scolaires ou bien l'inexistence d'une institution donnée dans un autre pays), ont amené le traducteur à formuler « une réalité ou un symbole donné [...] selon la conception et l'organisation du mode de vie (et de pensée) d'une communauté linguistique déterminée » (Bastin, 1993: 475). L'application du procédé adaptatif est née du souci d'établir une équivalence de sens entre les textes source et cible et par conséquent, de mieux faire comprendre le texte d'arrivée aux spectateurs étrangers. Or, dans le cadre des JT d'Arte, l'emploi de ce procédé demeure marginal.

Par contre, en basant sur les versions doublées (française, polonaise et allemande) du film américain Shrek, on a découvert que les traducteurs audiovisuels se servaient relativement souvent des techniques adaptatrices afin de rendre compréhensibles les éléments appartenant à une socioculture donnée aux spectateurs ressortissant de différents contextes culturels et langagiers ${ }^{4}$ On est d'avis que deux prémisses sont à l'origine de l'emploi des techniques adaptatrices dans les versions doublées du film américain :

Premièrement, pour faire également rire le public d'arrivée, le traducteur introduit dans les dialogues filmiques toute sorte d'éléments propres à la culture cible qui déclenchent le rire et l'amusement chez les spectateurs d'arrivée. Le but principal consiste à produire le même effet comique.

Deuxièmement, le recours aux techniques adaptatrices et par conséquent, la facilité d'accès à ses propres allusions résultent du fait que dans la traduction audiovisuelle où il n'y a pas de retour en arrière, ni d'astérisque, ni de notes au bas de page, c'est la rapidité de transmission du message qui compte le plus. Et ce transfert rapide du message médiatique implique aussi la rapidité de la réception de celui-ci par les spectateurs. Ainsi, en utilisant le procédé d'adaptation, les traducteurs peuvent rapidement produire le même effet chez les récepteurs des films étrangers. Cet accès facile et rapide à l'humour contenu dans les dialogues filmiques rend le film plus attractif et populaire. Faute de temps pour expliquer les éléments étrangers dans un film doublé, les traducteurs recourent au procédé d'adaptation et cherchent dans les cultures cibles les éléments connus, facilement déchiffrables par les publics d'arrivée qui déclencheraient les mêmes réactions que celles chez le public de départ. On a donc affaire à des traductions qui visent à être comprises rapidement, immédiatement et qui sont amusantes pour chaque spectateur quel que soit son âge. Par conséquent, en regardant les versions doublées de Shrek qui sont toutes traduites de l'anglais, les spectateurs d'arrivée peuvent parfois avoir l'impression que le film est ancré dans la réalité qu'ils connaissent bien et

${ }^{4}$ Pour plus de détails sur ce sujet, on invite à lire notre article Les techniques adaptatrices utilisées par les traducteurs audiovisuels dans les versions doublées (française, polonaise et allemande) du film americain Shrek paru dans «Francophonie et interculturalite " sous la direction de J. Lis et T. Tomaszkiewicz (2008), Oficyna Wydawnicza LEKSEM, Łask. 
qu'il a été crée pour eux. Il faut tout de suite mentionner que c'est le doubleur polonais qui aurait tendance à employer plus souvent les techniques adaptatrices que les traducteurs français et allemand. Et même, il les introduira là où il n'y en a ni dans l'original, ni dans d'autres versions doublées. Passons donc aux exemples concrets :

\section{- L'adaptation des noms propres}

L'adaptation des noms propres consiste en remplacement d'un prénom original par un prénom caractéristique en langue d'arrivée. Ce procédé qui est surtout observable dans la traduction polonaise a pour but de donner l'impression au spectateur d'arrivée qu'il s'agit d'un film ancré dans la réalité polonaise et par conséquent, d'obtenir un effet comique. En voici les exemples :

\begin{tabular}{|l|l|l|l|}
\hline \multicolumn{1}{|c|}{ Texte anglais (TD) } & \multicolumn{1}{|c|}{ Texte français (TA) } & Texte allemand (TA) & \multicolumn{1}{c|}{ Texte polonais (TA) } \\
\hline $\begin{array}{l}\text { - So I see. Let's go } \\
\text { Kyle. }\end{array}$ & $\begin{array}{l}\text { - Oui, je vois ça. Allez, } \\
\text { en route Kyle. }\end{array}$ & - Fahren wir, Kyle. & $\begin{array}{l}\text { - Wlaśnie widzę. } \\
\text { Jedziemy panie Zdzisiu. }\end{array}$ \\
\hline $\begin{array}{l}\text { - There you go, boys. } \\
\text { - Just leave the bottle, } \\
\text { Doris. }\end{array}$ & $\begin{array}{l}\text { - Je vous sers, les gars. } \\
\text { - Laissez-nous la } \\
\text { bouteille, Doris. }\end{array}$ & $\begin{array}{l}\text { - Bitte sehr, Jungs. } \\
\text { - Lass die Flasche da, } \\
\text { Doris. }\end{array}$ & $\begin{array}{l}\text { - Na pohybel władzy. } \\
\text { - Zostaw cała, Wojtuś. }\end{array}$ \\
\hline $\begin{array}{l}\text { - Is that you, Gorder? } \\
\text { - How did you know? }\end{array}$ & $\begin{array}{l}\text { - Baisse-toi, Gorder. } \\
\text { - Comment tu as } \\
\text { deviné? }\end{array}$ & $\begin{array}{l}\text { - Bist du das, Gorder? } \\
\text { - Woher weisst du } \\
\text { das ? }\end{array}$ & $\begin{array}{l}\text { - Zygmunt, to ty? } \\
\text { - A kto ma być? }\end{array}$ \\
\hline
\end{tabular}

Dans tous les trois exemples on peut observer que seulement le traducteur polonais a recouru à l'adaptation. Le premier cas concerne un prénom méconnu en Pologne Kyle. Dans cette scène il est question d'une relation employeur - employé (Marraine la Bonne Fée et son chauffeur) qui en polonais est exprimée par une formule de politesse très fréquemment utilisée dans ce type de relations interpersonnelles (pani / panie (Madame / Monsieur) + prénom, ici panie Zdzisiu).

Le deuxième cas qui prend la forme d'un diminutif, se réfère à la personne du fameux journaliste et présentateur polonais Wojciech Mann qui prête sa voix au personnage de l'Affreuse belle-sœur dans le film.

Dans le dernier exemple, c'est aussi uniquement le traducteur polonais qui a décidé de remplacer le prénom Gorder par le prénom polonais Zygmunt. Le fait d'appeler une souris par un prénom attribué à un être humain donne un effet humoristique. Pourtant, on peut remarquer que dans les films doublés en français et en allemand où le prénom original Gorder a été laissé tel quel, les souris parlent avec un accent américain, ce qui certainement fait rire les publics d'arrivée. 


\begin{tabular}{|l|l|l|l|}
\hline \multicolumn{1}{|c|}{ Texte anglais (TD) } & \multicolumn{1}{|c|}{ Texte français (TA) } & \multicolumn{1}{c|}{ Texte allemand (TA) } & \multicolumn{1}{c|}{ Texte polonais (TA) } \\
\hline - Now, ye ogre, pray & - A nous deux, l'ogre ! & - Und jetzt, du Oger, & - Jam Puszek Okruszek, \\
for mercy from... & Crains mon P vengeur. & bitte um Gnade vom... & kto ze mną zadrze... \\
Puss... in Boots $!$ & Je suis le Chat... Potté. & Gestifelten Kater. & umarł w butach. \\
\hline
\end{tabular}

Dans l'exemple suivant, il est question du personnage provenant du conte de Charles Perrault Le chat botté. Dans les traductions française et allemande les traducteurs se sont servis d'un équivalent culturel propre à ces deux langues, notamment le chat botté et der gestifelte Kater. Or, comme on peut l'observer et ce n'est pas une faute, on retrouve dans le texte français le nom du chat dont l'ortographe change. Tout simplement parce que le chat lors de l'énonciation de ces paroles a tracé la lettre P sur un tronc d'arbre à l'instar de Zorro. Ainsi, pour rester conforme à l'image, le traducteur français a un peu modifié l'ortographe du mot en question. Par contre, l'équivalent allemand et l'ortographe de ce mot ne correspondent pas à ce que l'on voit sur l'écran. On voit un chat botté mais on ne sait pas pourquoi il dessine la lettre $P$ sur le tronc d'arbre.

Par contre, le traducteur polonais, au lieu de recourir à l'appellation équivalente du chat botté (en polonais : kot w butach) où il n'y a pas de lettre $p$ au début des mots, a fait référence au personnage mentionné dans une chanson polonaise, à savoir Puszek Okruszek. Puszek Okruszek est un chien, mais ici, on applique ce nom à un chat, et ceci pour obtenir l'effet conforme aux images filmiques.

\section{- Référence à la cuisine}

Il est évident que chaque pays a ses plats traditionnels qui sont méconnus dans d'autres pays et par conséquent, les noms de ces plats ne possèdent pas d'équivalents lingustiques et culturels dans la langue et culture réceptrice. Dans ce cas-là, pour rendre compréhensible le message d'arrivée, le traducteur peut se servir des méthodes d'adaptation.

\begin{tabular}{|l|l|l|l|}
\hline \multicolumn{1}{|c|}{ Texte anglais (TD) } & \multicolumn{1}{|c|}{ Texte français (TA) } & Texte allemand (TA) & \multicolumn{1}{|c|}{ Texte polonais (TA) } \\
\hline - You know what else & - Tu sais ce que tout le & - Weisst du, was alle & - Wiesz co jeszcze \\
everybody like? & monde aime ? Le & noch mögen? Parfait. & wszyscy lubią? Kre- \\
Parfaits. Have you ever & clafoutis. T'as dejà & Hast du je einen & mówki. Znasz kogoś, \\
met a person, you say, & rencontré quelqu'un à & getroffen, der sagt: & komu jak powiesz: \\
"Hey, let's get some & qui tu dis : « Fais péter & "Parfait mag ich & "Chodź na kremówki, \\
parfaits", they say, & le clafoutis », et qui te & nicht“? Parfaits sind & mówi „Nie stary, nie \\
"Hell, no, I don't like & dit, « J'aime pas le & wohl die leckerste & lubię kremówek”. \\
no parfaits"? Parfaits & clafoutis »? Le & Sache aud dem ganzen & Kremówki są pycha. \\
are delicious. & clafoutis, c'est exquis. & Planeten. & \\
\hline
\end{tabular}

Dans la version originale, on emploie le mot parfaits pour désigner une sorte de dessert, notamment une glace enrobée de diverses couvertures, comme p.ex. de 
la crème fouettée et des fruits. Pourtant, même si ce mot qui existe aussi en français et qui se réfere soit à la glace qui ne contient que de la crème fraîche et d'un parfum, soit au nom du fromage, le traducteur a préféré le remplacer par le nom du gâteau français le clafoutis qui se fait à base de farine, de lait, d'œufs et de fruits mêlés (p.ex. le clafoutis aux cerises). En ce qui concerne le texte allemand, le traducteur a gardé le nom original de cette glace qui est également connue et servie en Allemagne comme dessert. En plus, en Allemagne cet emprunt à la langue française renvoie aussi au nom du pâté à tartiner fait à base de viande ou de poisson.

Quant à l'adaptation polonaise le mot parfaits a été remplacé par le nom du gâteau polonais kremówki. Il existe une petite histoire qui a rendu célébre ce nom du gâteau polonais : le pape Jean Paul II a prononcé la phrase suivante lors de sa visite en Pologne dans la ville natale en 1999: A po maturze chodziliśmy na kremówki (Après le bac, nous allions manger kremówki). Ses paroles sont très vite devenues célèbres et ont été citées dans tous les médias polonais.

On peut observer que dans les deux traductions : française et polonaise, on a effectivement affaire à l'adaptation du terme étranger.

Introduction des éléments propres à la socioculture polonaise

Dans le film Shrek on peut aussi trouver des fragments où le traducteur polonais introduit les éléments culturels dans le texte cible même s'ils sont absents dans le texte original. Il en est question dans les exemples suivants :

\begin{tabular}{|l|l|l|l|}
\hline \multicolumn{1}{|c|}{ Texte anglais (TD) } & \multicolumn{1}{|c|}{ Texte français (TA) } & Texte allemand (TA) & \multicolumn{1}{|c|}{ Texte polonais (TA) } \\
\hline - Donkey, what are you & - L'Ane, qu'est-ce que & - Esel, was machst du & - Ośle, móglbym \\
doing here? & tu fais ici ? & hier? & wiedzieć, co ty robisz? \\
- Taking care of your & - J'suis venu garder & - Mich um euer & - Zajmowalem się \\
love nest for you. & votre nid d'amour. & Liebesnest kümmern. & waszym M4, a co? \\
\hline
\end{tabular}

Dans ce cas, on trouve dans la traduction polonaise, l'expression M4 qui désigne un appartement de trois pièces, une cuisine et une salle de bains, appelé ainsi à l'époque du communisme. Par contre, dans les textes français et allemand, les traducteurs ont employé un équivalent lexical du terme anglais love nest, notamment le nid d'amour et das Libesnest.

Pour conclure notre article sur l'emploi de la démarche adaptative dans l'audiovisuel, on tient à remarquer que :

1. Aussi bien dans les JT d'Arte que dans les versions doublées du Shrek, l'utilisation de la démarche adaptative comprise comme un procédé de traduction ponctuel ne concerne pas tout le texte mais porte sur certains fragments du discours médiatique.

2. Dans le cas des JT d'Arte qui servent à présenter les événements tels quels sont en réalité, l'emploi du procédé d'adaptation est peu fréquent, marginal et non abusif. 
3. Pourtant, en ce qui concerne les versions doublées du film américains Shrek qui est un film de fiction, les traducteurs recourent plus souvent et plus volontièrement à l'usage des techniques adaptatrices pour faire rire les publics d'arrivée. Et même si le procédé d'adaptation est parfois perçu comme résultat de l'inéfficacité du transfert du culturel et même si en l'utilisant, on gomme complétement les éléments socioculturels étrangers, on ne peut pas nier le fait que dans le cas des films d'animation, il contribue à l'augmentation de la popularité des films doublés et constitue l'une des modalités de transmission du sens dans l'acte traduisant.

\section{BIBLIOGRAPHIE}

Bastin G.L. (1990), « Traduire, adapter, réexprimer ", Meta, 35.

Bastin G.L. (1993), « La notion d'adaptation en traduction », Meta, 38, p. 473-478.

Bensimon, P. (1998), «Présentation », Palimpsestes, XI, Traduire la culture, Paris : Presses de la Sorbonne Nouvelle.

Berman A. (1984), L'épreuve de l'étranger. Culture et traduction dans l'Allemagne romantique, Paris : Editions Gallimard.

Borowczyk P. (2004), Stratégies des traducteurs audiovisuels face aux realités socioculturelles sur l'exemple de la télévision Arte. Praca doktorska obroniona na Wydziale Neofilologii UAM.

Borowczyk P. (2008), «Les techniques adaptatrices utilisées par les traducteurs audiovisuels dans les versions doublées (française, polonaise et allemande) du film américain Shrek », in : Francophonie et interculturalité, dir. J. Lis et T. Tomaszkiewicz, Łask : LEKSEM.

Catford J.C. (1965), A Linguistic Theory of Translation, London : Oxford University Press.

Chaciński M. (2007), «Zagubieni w przekladzie », Przekrój nauki, 3, p. 44-46.

Dąbska-Prokop U. (red.) (2000), Mała encyklopedia przekładoznawstwa, Częstochowa : Educator.

Delisle J., Lee-Jahnke H., Cormier C.M. (1999), Terminologie de la traduction, Amsterdam-Philadelphia : John Benjamins Publishing Company.

Gambier Y, (1992), «Adaptation : une ambiguilté à interroger », Meta, 37.

Hurtado-Albir A. (1990), La notion de fidélité en traduction, Paris : Didier Erudition.

Kielar B. (1988), Ttumaczenie i koncepcje translatoryczne, Wrocław : Ossolineum.

Koller W. (1992), Einführung in die Übersetzungswissenschafi, Heidelberg-Wiesbaden: Quelle und Meyer.

Ladmiral J.-R. (1998), «Le prisme interculturel de la traduction », Palimpsestes XI, Traduire la culture, Paris : Presses de la Sorbonne Nouvelle.

Lécrivain C. (1998), «Europe, traduction et spécificités culturelles », in : Europe et traduction, dir. M. Ballard, Artois : Artois Presses Université.

Lederer M. (1994), La traduction aujourd hui, Modèle interprétatif, Paris : Hachette.

Luyken G-M. et al. (1991), Vaincre les barrières linguistiques à la télévision. Doublage et sous-titrage pour le public européen, Manchester : Institut Européen de la Communication.

Nida E. (1964), Towards the Science of Translating, Leiden : Brill.

Pisarska, A., Tomaszkiewicz, T. (1996), Wspótczesne tendencje przekładoznawcze, Poznań : Wydawnictwo Naukowe UAM.

Reiss K., Vermeer H.J. (1991), Grundlegung einer allgemeinen Translationstheorie, Tübingen : Niemeyer. 
Stolze R. (1997), Übersetzungstheorien ; Eine Einführung, Tübingen : Gunter Narr Verlag.

Tomaszkiewicz T. (1993), Les opérations linguistiques qui sous-tendent le processus de sous-titrage des films, Poznań : Wydawnictwo Naukowe UAM.

Tomaszkiewicz T. (1998), «Traduction dans les mass-médias ", Scripta. Manent, Poznań : Wydawnictwo Wydziału Neofilologii.

Tomaszkiewicz T. (2006), Przektad audiowizualny, Warszawa : Wydawnictwo Naukowe PWN.

Vinay J.-P., Darbelnet J. (1966), Stylistique comparée du français et de l'anglais, Paris : Didier.

Wierzbięta B. (2004), «Co ja tu robię ? », Polityka, 27, p. 55.

Jarmołowicz J. (2004), «Specyfika przekładu utworów dramatycznych na podstawie wybranych węgierskich dramatów współczesnych », in: Hungarologia : jezzyk $i$ kultura, dir. I. Koutny, Prodruk, p. 89-119.

Sites Internet:

http://fr. wikipedia.org/wiki/Doublage (consulté le 02.09.2007)

http://urbanski.terramail.pl/strony/alex l.htm (consulté le 12.04.2007) 